\title{
Análisis de la Escala de Soledad de De Jong Gierveld mediante el modelo de Rasch*
}

\section{Analysis of the De Jong Gierveld Loneliness Scale Using the Rasch Model}

Recibido: enero 18 de 2012 | Revisado: julio 10 de 2012 | Aceptado: diciembre 22 de 2012

\author{
José BuZ** \\ Gerardo Prieto AdÁNeZ **** \\ Universidad de Salamanca, España
}

doi:10.11144/Javeriana.UPSY12-3.aesd

Para citar este artículo: Buz, J. \& Prieto, G. (2013). Análisis de la Escala de Soledad de De Jong Gierveld mediante el modelo de Rasch. Universitas Psychologica, 12(3), 971-981. doi:10.11144/Javeriana. UPSY12-3.aesd

* Artículo original de investigación. Agradecimientos: Este trabajo de investigación ha sido apoyado parcialmente por el proyecto AP-2004-5285 del Ministerio de Educación del Gobierno de España y el proyecto SA015A-08 concedido por la Consejería de Educación y Cultura, de la Junta de Castilla y León, España.

** Universidad de Salamanca. Dpto. de Psicología Evolutiva y de la Educación. Facultad de Educación. Paseo de Canalejas 169, 37008 Salamanca, España. E-mail: buz@usal.es

**** Universidad de Salamanca. Dpto. de Psicología Básica, Psicobiología y Metodología de las Ciencias del Comportamiento. Facultad de Psicología. Avda. de la Merced 109, 131. 37005 Salamanca, España. E-mail: gprieto@usal.es

\section{RESUMEN}

Este trabajo examina mediante el modelo de Rasch las propiedades psicométricas de la adaptación española de la escala de Soledad de De Jong Gierveld. Participaron 614 personas (60-96 años) no institucionalizadas. También se midieron los afectos (PANAS) y la satisfacción con la vida. Los valores del ajuste al modelo y la precisión de las medidas de los ítems fueron elevados (0.99). La fiabilidad de las puntuaciones de las personas (0.69) y los ítems (0.77) fue baja. Los análisis factoriales confirmaron una estructura unidimensional. Algunos ítems mostraron un funcionamiento diferencial (FDI) asociado al sexo y a la forma de vida. Las garantías sobre la validez convergente/discriminante fueron moderadas. Los resultados deberían ser tomados en consideración para una revisión del instrumento.

\section{Palabras clave autores}

Rasch, validez, fiabilidad, soledad, personas mayores.

Palabras clave descriptores

Psicometría, validez, fiabilidad, España.

\section{A B S T R A C T}

This study examines the psychometric properties of the Spanish adaptation of the De Jong Gierveld Loneliness Scale by means of the Rasch model. Participants were 614 Spanish community-dwelling older adults (60-96 years). Convergent/divergent validity was examined by correlating the scale with both the PANAS scale and a satisfaction with life scale. Results showed that our data fit the model and that average item reliability were high (0.99), but average person reliability was low (0.69). Factorial analysis confirmed a unidimensional structure. Some items had a significant DIF for sex and living arrangements. The scale demonstrated moderate convergent/discriminant validity. These results should be taken into account for a revision of the instrument.

Key words authors

Rasch, validity, reliability, loneliness, older adults.

Key words plus

Psychometry, validity, reliability, Spain. 
La soledad es una experiencia subjetiva y desagradable resultado de la percepción de unas relaciones sociales deficitarias (De Jong Gierveld, 1987). Se ha asociado positivamente con problemas de salud mental (Losada et al., 2012; Prieto, Forjaz, Fernández, Rojo \& Martínez, 2011), los afectos negativos (Dykstra, 2009), el riesgo de enfermedades y la mortalidad (Tilvis, Laitala, Routasalo \& Pitkälä, 2011) y, negativamente, con la satisfacción con la vida (Victor, Scambler, Bond \& Bowling, 2000). Por ello, su medición forma parte de la evaluación de la calidad de vida y del bienestar en la vejez (Walker, 2005).

A pesar de su importancia, se carece de instrumentos de medición validados en la población mayor española. Los instrumentos existentes tienen su origen en culturas individualistas muy distintas a la cultura colectivista española. Por tanto, algunos aspectos de su validez pueden ser cuestionados. El objetivo de este trabajo fue examinar las propiedades psicométricas de la escala de soledad de De Jong Gierveld (De Jong Gierveld \& Kamphuis, 1985) mediante el modelo de Rasch, en una muestra de personas mayores españolas. En España, los estudios psicométricos de esta escala han sido escasos (p. ej., Sánchez, 2009), y ninguno con el modelo de Rasch desde el que fue creada.

\section{La Escala de Soledad de De Jong Gierveld}

Esta escala se fundamenta en el modelo cognitivo de la soledad de De Jong Gierveld (1987). Según el modelo, cuanto mayor es la discrepancia entre las relaciones sociales deseadas y las que efectivamente se tienen, mayor es la experiencia de soledad. En la última década, la escala de Soledad de De Jong Gierveld (De Jong Gierveld \& Kampuis, 1985) ha sido probablemente la escala más utilizada en Europa para medir este constructo. De ella se ha destacado que está fundamentada en un modelo teórico, que es breve, que ha sido utilizada en numerosos países y que no presenta sesgo debidos a la cultura (Scharf \& De Jong Gierveld, 2008). Según los autores, el propósito es medir el grado de soledad que experimenta una persona en una dimensión básica. No obstante, sus ítems tienen en cuenta la distinción hecha por Weiss (1973) sobre la soledad emocional y social. Seis ítems miden la soledad emocional derivada del abandono o ausencia de personas queridas. Los otros cinco ítems miden la soledad social producida por el deseo de contar con alguien en caso de necesidad.

Los autores de la escala han defendido insistentemente su estructura unidimensional (para una revisión, véanse De Jong Gierveld \& Van Tilburg, 2011), aunque han reconocido carencias en su homogeneidad y en la escalabilidad de los sujetos ( $\mathrm{p}$. ej., Van Tilburg \& De Leeuw, 1991). La consistencia interna de la escala (alfa de Cronbach o rho) ha oscilado entre 0.81 y 0.9 (Bartlett, Warbuton, Lui \& Peach, 2008; Van Baarsen, Snijders, Smit \& Knipscheer, 1999; Van Tilburg \& De Leeuw, 1991). La aparición, en algunos estudios, de dos factores asociados a la soledad social y emocional ha sido atribuida a un sesgo de método, afirmándose que "no había una base teórica para la bidimensionalidad" (De Jong Gierveld \& Kamphuis, 1985, p. 295). Los resultados de sus investigaciones en Canadá, Italia y Holanda apuntan en este mismo sentido (Van Tilburg, Havens \& De Jong Gierveld, 2004).

Por el contrario, otros estudios han considerado que la escala era bidimensional. Por ejemplo, Van Baarsen et al. (1999) encontraron dos factores con valores de homogeneidad modestos y una moderada correlación entre sí. El primero, de soledad emocional, estaba formado por los seis ítems formulados negativamente y el segundo, de integración social, por los cinco ítems restantes. Ambos factores explicaban el $53.8 \%$ de la varianza total. Posteriormente, Van Baarsen, Snijders, Smit y Duijn (2001) afirmaron que la escala no cumplía criterios de unidimensionalidad según el modelo de Rasch, además de existir sesgo de género en los ítems. Los resultados de Zammuner (2008) y de otros autores con población joven y adulta (p. ej., Kunts, Bogaerts \& Winkel, 2010) también apoyan la bidimensionalidad de la escala.

La escala ha sido ampliamente utilizada en diferentes países, mostrando en general unas garantías psicométricas adecuadas. Sin embargo, la experiencia de soledad está muy influida por la cultura (Rokach, Orzeck, Cripps, Lackovic-Grgin \& 
Penezic, 2001). En el presente estudio se consideró necesario examinar la generalización de la validez de la Escala de Soledad de De Jong Gierveld, ya que esta fue creada en una cultura individualista donde los estándares de las relaciones interpersonales y las normas de cuidados son distintos a los de la cultura colectivista (Hank, 2007). Por ejemplo, en España, vivir solo tiene connotaciones de abandono y desamparo familiar (Lorenzo \& Castejón, 2008). Por el contrario, en Holanda, vivir solo es un indicador de competencia y autonomía personal (Scharf \& De Jong Gierveld, 2008). Quizás por ello se ha encontrado que en España las personas mayores experimentan niveles de soledad más altos que sus iguales en Holanda (Sánchez, De Jong Gierveld \& Buz, 2012).

La diferencia cultural en las creencias asociadas a la forma de interacción social y a la forma de vida podría implicar la presencia de sesgo en los ítems. Por ello, en este trabajo además de examinar el ajuste de los datos al modelo de Rasch, se comprobó la invarianza métrica de los ítems (una condición de la validez generalizada) entre hombres y mujeres, y entre las personas que vivían solas o acompañadas. Esto es especialmente importante si se quieren comparar los efectos diferenciales de la ausencia de seres queridos sobre la soledad. También se examinó la validez externa mediante la asociación de la soledad con los afectos positivos y negativos y la satisfacción con la vida.

\section{Método}

\section{Participantes}

En este estudio participaron 614 personas mayores de 60 años $(M=74.05 ; D E=7.7$; Rango $=60$ 96), no institucionalizadas. Se realizó un muestreo incidental, estratificado por edad y sexo, en cinco provincias de la Comunidad de Castilla y León. El $54 \%$ de los participantes fueron mujeres y el estado civil más frecuente fue el de casado (52.4\%). La mayoría vivía con el cónyuge y/o los hijos (69.4\%). La mayor parte de ellos (73.6 \%) tenían estudios primarios y/o secundarios, y solo un $7.2 \%$ había ido a la universidad. El 86.6 \% podía hacer tareas cotidianas de forma autónoma. El perfil sociodemográfico de la muestra era comparable con el perfil general de la población mayor española (Instituto de Mayores y Servicios Sociales [IMSERSO], 2008).

\section{Instrumentos}

Se utilizó la Escala de Soledad de De Jong Gierveld (De Jong Gierveld \& Kamphuis, 1985) de 11 ítems con tres categorías de respuestas $(1=$ no, 2 = más o menos, $3=$ sí). Los ítems se refieren a si las personas experimentan situaciones donde el número de relaciones interpersonales es más pequeño del deseado (p. ej., "Tengo mucha gente en la que confiar plenamente") o si se ha alcanzado el nivel deseado de intimidad (p. ej., "Echo de menos tener un buen amigo de verdad"). Siguiendo las instrucciones de los autores, la puntuación de soledad se obtiene dicotomizando las respuestas, de modo que se obtiene un punto si se responde más o menos o no en los ítems 1, 4, 7, 8 y 11. En los restantes ítems se obtiene un punto si se responde más o menos o sí. La puntuación final oscila entre 0 (No soledad) hasta 11 (Soledad extrema), resultado de sumar el número de respuestas afirmativas o negativas en todos los ítems.

Para minimizar las fuentes de error en la adaptación de la escala (Muñiz, 2004), se usó un sistema de doble traducción (holandés-español-holandés) con dos traductores bilingües de lengua materna holandesa y española, respectivamente. La traducción final al castellano se consensuó entre dos psicólogos expertos en el tema de estudio y los investigadores. El ítem 10 ("Vaak voel ik me in de steek gelaten" [Se siente abandonado a menudo]) fue traducido en el sentido de "abandonado" en lugar de "rechazado", ya que se ajustaba mejor al significado connotativo original y al de otras traducciones (francés e italiano). Igualmente, en el ítem 6 ("Ik vind mijn kring van kennissen te beperkt" [Piensa que su círculo de amistades es demasiado limitado]) el término kennissen fue traducido como "amistades" en lugar de su traducción más literal de "conocidos". En la versión en inglés de la escala los autores incluyen en el enunciado a los amigos y conocidos, pero se consideró que se trata de dos 
tipos de relaciones muy distintas que no deberían usarse como sinónimos en un mismo enunciado.

Se midieron los afectos con la Escala de Afectos Positivos y Negativos (Positive and Negative Affect Schedule [PANAS]; Watson, Clark \& Tellegen, 1988 adaptación española de Sandín et al., 1999). Desde el modelo teórico original, la soledad se asocia al afecto negativo (Dykstra, 2009). La escala se compone de 20 adjetivos (10 positivos y 10 negativos) que describen experiencias afectivas (p. ej., animado, culpable) a las que el sujeto debe responder según el grado en que las ha experimentado a lo largo de las últimas semanas utilizando para ello una escala de 1 (nada) a 5 (mucho). Dada la naturaleza ordinal de los ítems, la asimetría de las distribuciones y la alta variabilidad en los pesos factoriales detectada en análisis preliminares, se calculó la consistencia interna desde una aproximación no lineal, usando el procedimiento implementado por Mislevy y Bock (1990). Esta fiabilidad se estimó para el afecto positivo (AP) y el afecto negativo (AN) en 0.88 y 0.86 , respectivamente.

Por último, se empleó la Escala de Satisfacción con la Vida (Satisfaction with Life Scale [SWLS]; Diener, Emmons, Larsen \& Griffin, 1985 adaptación española de Pons, Atienza, Balaguer \& GarcíaMerita, 2002). Según sus autores, esta escala mide el componente cognitivo del bienestar subjetivo y no se solapa con otros constructos, como los afectos o la soledad (p. 71). Se compone de 5 ítems a los que el sujeto responde según su grado de acuerdo en una escala que va de 1 (totalmente en desacuerdo) a 5 (totalmente de acuerdo). La consistencia interna, aplicando el procedimiento anterior, se estimó en 0.79 .

\section{Procedimiento}

La escala de soledad se aplicó en el contexto de un estudio más amplio que incluía distintas variables sobre el apoyo social en la vejez. La recogida de datos se realizó contactando con las personas a través de centros educativos para mayores, centros de ocio y parroquias. Tras un primer contacto, se obtuvo el consentimiento verbal y se concertó una entrevista en el hogar. La aplicación de la escala se realizó mediante entrevista oral por entrevistadores entrenados garantizando el anonimato y la confidencialidad de los datos. La participación fue voluntaria y no remunerada.

\section{Análisis de datos}

Se examinaron las garantías psicométricas de la escala mediante el modelo de Rasch, siguiendo las recomendaciones de Prieto y Delgado (2003). Según Embretson y Reise (2000), el modelo de Rasch presenta ventajas métricas sobre la TCT ya que permite comprobar: a) si las escalas tienen propiedades de intervalo, b) si los parámetros de los ítems y de las muestras son independientes entre sí, c) si los ítems de la escala miden unidimensionalmente el constructo de la soledad y d) si el instrumento cuenta con suficientes garantías de fiabilidad a nivel de ítems y de las personas.

Tras poner a prueba el grado de unidimensionalidad de la escala, se evaluaron la validez generalizada y la validez convergente y discriminante. Dado que se trata de una escala bidireccional, los ítems de soledad social fueron codificados de forma inversa. Para el análisis descriptivo de los datos y la validez convergente/discriminante se usó el programa SPSS 19 para Windows. Para evaluar la unidimensionalidad desde el modelo de Rasch se utilizó WINSTEPS (Linacre \& Wright, 2000), mientras que para obtener la consistencia interna de las escalas de afectos y de satisfacción con la vida y realizar los análisis factoriales no restrictos a partir de la comparación de los residuos estandarizados de matriz de correlaciones se utilizó FACTOR (Lorenzo-Seva \& Ferrando, 2006).

La Tabla 1 muestra los estadísticos de ajuste de los ítems (infit y outfit), la localización $\left(D_{i}\right)$, el error estándar (EE) y la correlación ítem-total. Los valores medios de infit y outfit ( 0.99 , en ambos casos) para el ajuste global de los ítems estaban muy cerca del valor de ajuste perfecto. El rango de valores de los infit (0.85-1.12) y de los outfit (0.63-1.58) indicaba un ajuste adecuado al modelo (Linacre $\&$ Wright, 2000). Los valores del ajuste global de las personas mostraron valores con una media y desviación estándar de 1 y 0.35 (infit), respectivamente y de 
TABLA 1

Propiedades psicométricas de los items

\begin{tabular}{lccccc}
\hline \multicolumn{1}{c}{ Ítems } & Infit & Outfit & $D_{i}$ & $E E$ & $R_{i x}$ \\
\hline 1-Siempre hay alguien con quien puede hablar de sus problemas diarios. (-) & 0.98 & 0.92 & 0.95 & 0.13 & 0.57 \\
2-Echa de menos tener un buen amigo de verdad. (+) & 1.12 & 1.13 & -1.54 & 0.1 & 0.5 \\
3-Siente una sensación de vacío a su alrededor. (+) & 0.85 & 0.72 & 0.22 & 0.11 & 0.63 \\
4-Hay suficientes personas a las que puede recurrir en caso de necesidad. $(-)$ & 0.88 & 0.63 & 2.04 & 0.17 & 0.59 \\
5-Echa de menos la compañía de otras personas. (+) & 0.91 & 0.85 & -1.9 & 0.1 & 0.58 \\
6-Piensa que su círculo de amistades es demasiado limitado. (+) & 1.04 & 1.05 & 0.6 & 0.1 & 0.54 \\
7-Tiene mucha gente en la que confiar completamente. (-) & 1.15 & 1.58 & -1.55 & 0.1 & 0.47 \\
8-Hay suficientes personas con las que tiene una amistad muy estrecha. $(-)$ & 1 & 1 & 1.42 & 0.14 & 0.55 \\
9-Echa de menos tener gente a su alrededor. (+) & 0.94 & 0.92 & -1.1 & 0.1 & 0.58 \\
10-Se siente abandonado a menudo. (+) & 0.97 & 0.72 & 0.97 & 0.13 & 0.58 \\
11-Puede contar con sus amigos siempre que lo necesita. (-) & 1.09 & 1.36 & 1.09 & 0.13 & 0.51 \\
\hline$M$ & 0.99 & 0.99 & 0 & 0.12 & \\
DE & 0.09 & 0.27 & 1.32 & 0.02 & \\
\hline
\end{tabular}

Nota. Los ítems marcados con (+) están en la dirección del constructo y miden el componente emocional de la soledad. Los ítems con (-) están en la dirección negativa y miden el componente emocional. $D_{i}=$ localización; $E E=$ error estándar; $R_{i \chi}=$ correlación ítem-total.

Fuente: elaboración propia.

0.99 y 0.95 (outfit), respectivamente. El número de personas desajustadas con valores de infit y/o outfit $>2$ fue bajo (9.04\%). Por tanto, se puede afirmar que las respuestas empíricas se ajustaban de manera aceptable a las predicciones del modelo.

En cuanto a la estimación de los parámetros de los ítems, la mayor parte de ellos presentó valores fiables, con errores estandar bajos (EE entre 0.1 y 0.17 ). Las correlaciones ítem-test eran altas y similares en magnitud, lo que indicaba un nivel de discriminación adecuado. La localización de los ítems en el continuo ( $\delta$ entre 2.04 y -1.9) permitía identificar un amplio rango de experiencias de soledad. El ítem 4 ( $\delta=2.04)$ resultó ser el más "difícil” (Tiene suficientes personas a las que recurrir en caso de necesidad) y el ítem $5(\delta=-1.9)$ era el más "fácil" (Echa de menos la compañía de otras personas). La estimación global de la fiabilidad de las medidas de los ítems mostró que la escala tenía una alta precisión (item separation reliabilitity $=0.99$ ).

Las estimaciones de los parámetros de los sujetos se distribuyeron en valores logits de entre 2.92 y -2.94, con una media y desviación estándar de -1.09 y 0.94, respectivamente. Los errores estándar oscilaron entre 0.72 y 1.88. La fiabilidad de las medidas de las personas se aproximaba al nivel mínimamente aceptable (person separation reliability $=0.69$ ). $\mathrm{La}$ fiabilidad de las puntuaciones directas fue algo más alta $(\alpha=0.77, \mathrm{KR}-20)$. El escalamiento conjunto de ítems y de personas (Figura 1) permitió observar que se trataba de una distribución asimétrica positiva, donde aproximadamente el $80 \%$ de las personas tenía puntuaciones logit por debajo de 0 , indicativas de bajos niveles de soledad.

Además de utilizar un contraste omnibus de los supuestos del modelo (infit y outfit), se analizó la unidimensionalidad de la escala mediante un análisis factorial exploratorio siguiendo las recomendaciones de Ferrando y Anguiano-Carrasco (2010). Debido a que los ítems estaban dicotomizados, se utilizó como input la matriz de correlaciones tetracóricas. Además, se empleó el método de extracción de mínimos cuadrados no ponderados ([ULS]; Jöreskog, 1977), ya que se trata del método más robusto para estas estimaciones (Abad, Olea, Ponsoda \& García, 2011). Para decidir cuántos factores retener, se utilizó la técnica del análisis paralelo.

Comprobada la adecuación de los datos (Bartlett $<0.001$ y $\mathrm{KMO}=0.78)$, el análisis paralelo recomendó extraer un único factor. Este factor 


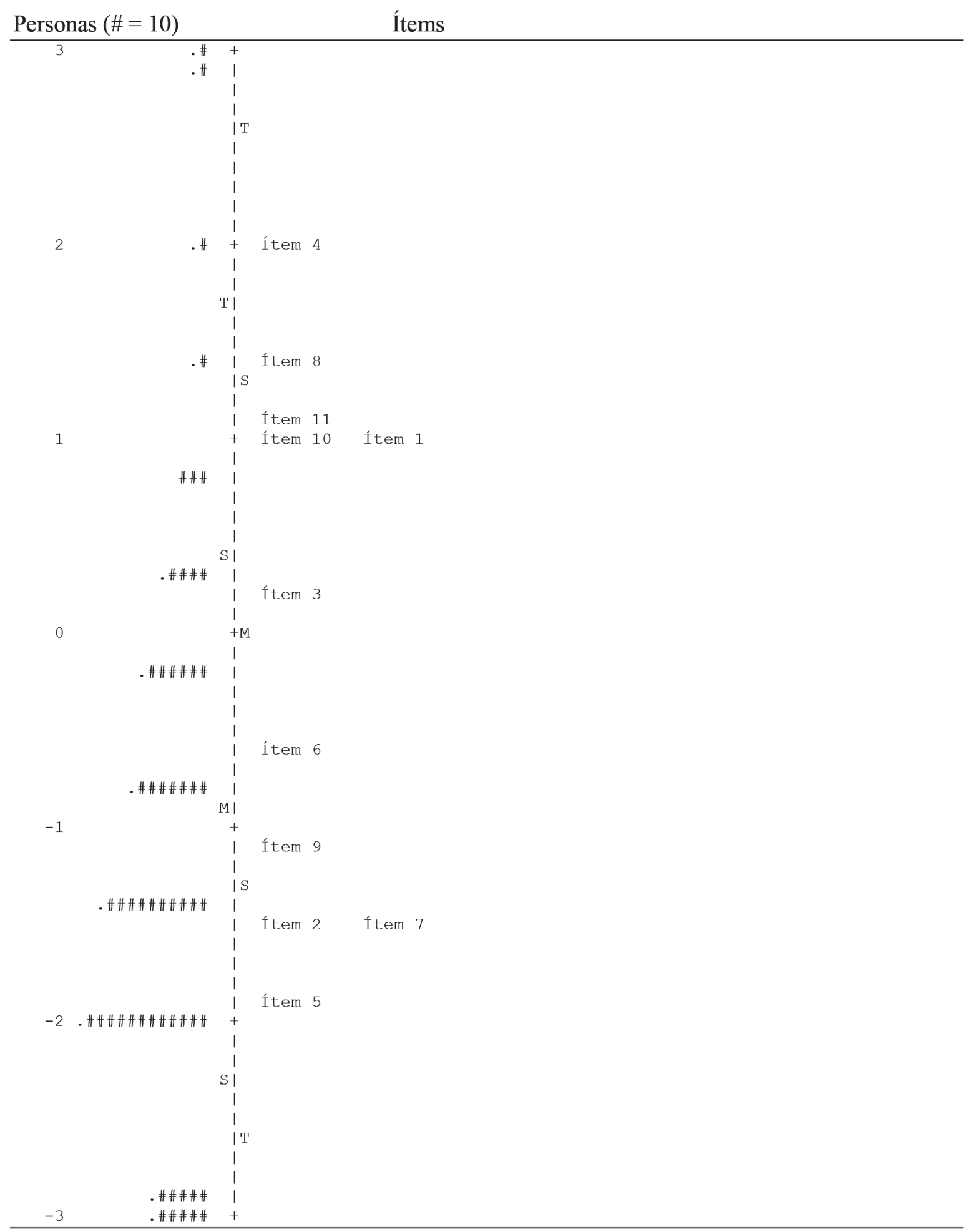

Figura 1. Mapa conjunto de personas e ítems a lo largo del continuo de la Soledad.

Nota. $\mathrm{M}=$ media, $\mathrm{S}= \pm 1$ desviación estándar y $\mathrm{T}= \pm 2$ desviaciones estándar de las personas y de los ítems. Fuente: elaboración propia. 
explicaba el $52.44 \%$ de la varianza, mientras que un segundo factor apenas explicaba el $10.44 \%$. El índice de bondad de ajuste absoluto de esta solución factorial fue bueno, GFI $=0.98$, así como la raíz media cuadrática residual, $\mathrm{RMCR}=0.09$, muy próxima al criterio de Hu y Bentler (1998) de 0.08. En consecuencia, se puede afirmar que la escala es fundamentalmente unidimensional.

Por último, para examinar si se podía generalizar la validez de la escala en función del sexo y de las condiciones de vida (vivir solo o acompañado), se analizó el funcionamiento diferencial de los ítems (FDI). La presencia de FDI mediante el modelo de Rasch se pone a prueba calculando la diferencia entre los estimadores del parámetro de dificultad del ítem en cada grupo (por ejemplo, varones y mujeres), controlando las posibles diferencias entre esos grupos en la variable latente. Wright y Douglas (1976) encontraron que las diferencias inferiores a 0.5 logit tenían consecuencias despreciables en la validez de las medidas. Por tanto, suele considerarse que el FDI es sustancial si la diferencia absoluta es superior a 0.5 logit y es estadísticamente significativa. Para contrastar la significación, se utiliza un contraste $t$ de Welch con la corrección de Bonferoni (Benjamini \& Hochberg, 1995). La presencia de FDI permitiría identificar factores ajenos al constructo medido que afectan inadecuadamente a las puntuaciones del test. Suele recomendarse la eliminación de los ítems con DIF por su falta de validez generalizada, especialmente cuando las puntuaciones en un test van a servir para tomar decisiones de importancia (Prieto \& Delgado, 2010).

Los análisis del FDI asociado al sexo revelaron dos ítems problemáticos: el ítem 2 (Echa de menos un amigo de verdad) era más fácil para los hombres $(t=-5.47, p<0.001$ ) y el ítem 3 (Siente una sensación de vacío alrededor) era más fácil para las mujeres $(t=4.42, p<0.001)$. Por otra parte, tras los análisis del FDI asociado al modo de vida se encontró que ocho de los once ítems (ítems 2, 3, 5, 6, 7, 9,10 y 11) presentaban propiedades psicométricas distintas según las condiciones de vida. En concreto, para las personas que vivían solas los ítems más fáciles eran: el ítem 3 (Siente una sensación de vacío alrededor) $(t=-5.76, p<0.001)$, ítem 5 (Echa de menos la compañía de otras personas $)(t=-2.97$, $p<0.003$ ), ítem 9 (Echa de menos tener gente alrededor) $(t=-2.94, p<0.003)$ y el ítem 10 (Se siente abandonado a menudo) $(t=-8.58, p<0.001)$. Sin embargo, para las personas que vivían acompañadas las respuestas más fáciles hacían referencia a las relaciones de amistad: ítem 2 (Echo de menos tener un buen amigo de verdad) $(t=4.66)$, ítem 6 (Pienso que mi círculo de amistades es demasiado limitado) $(t=4.97)$, ítem 7 (Tengo mucha gente en la que confiar completamente) $(t=3.6)$ e ítem 11 (Puedo contar con mis amigos siempre que lo necesito) ( $t=3.48, p<0.001$ en todos los casos).

Por último, en el examen de la validez convergente/discriminante las puntuaciones de la escala mostraron una asociación positiva con el afecto negativo, $r=0.31$ y una asociación negativa con el afecto positivo, $r=-0.3$, y con la satisfacción con la vida, $r=-0.32$ ( $p<0.001$, en todos los casos).

El objetivo principal de este estudio fue examinar, mediante el modelo de Rasch, las características psicométricas de las puntuaciones de la adaptación española de la Escala de Soledad de De Jong Gierveld. Para ello, se analizó el grado de ajuste de los ítems y las personas al modelo, y la precisión y distribución de las estimaciones de los parámetros. Los estadísticos referidos a los ítems y a las personas mostraron un adecuado ajuste de los datos al modelo y los patrones de respuestas de las personas se ajustaban a las predicciones de este. Los ítems presentaron niveles de discriminación adecuados y una alta precisión de sus parámetros. Sin embargo, las puntuaciones de las personas presentaron un insuficiente nivel de fiabilidad, probablemente debido al escaso número de ítems del instrumento. Aunque el valor KR-20 resultó ligeramente superior a 0.7 , y similar al encontrado en otros estudios (Dykstra, Van Tilburg \& De Jong Gierveld, 2005; Sánchez et al., 2012), hay que tener en cuenta que, en general, estos estadísticos de consistencia interna sobreestiman la fiabilidad (Abad et al., 2011). Parece necesario, por tanto, añadir más ítems para diferenciar adecuadamente a las personas con distintos niveles de soledad.

Los estadísticos de ajuste de los ítems y de las personas apoyaron la hipótesis de que los datos se 
ajustaban razonablemente al modelo, tal y como ha sido defendido por Moorer y Suurmeijer (1993) y los autores de la escala en distintas ocasiones (para una revisión véase, De Jong Gierveld \& Van Tilburg, 2011). A priori, los resultados convergen con los de Van Tilburg (1988) quien afirmó que la escala permite identificar un único rasgo latente que engloba componentes sociales y emocionales producto de la falta de relación con distintos tipos de personas. También se examinó desde un perspectiva factorial si las puntuaciones en los ítems de soledad social y emocional justificarían la existencia de una segunda dimensión en la escala como han encontrado otros autores (p. ej., Van Baarsen et al., 1999; Van Baarsen et al., 2001). A nuestro juicio, los resultados obtenidos no aportan suficientes evidencias para rechazar la hipótesis de unidimensionalidad. En todo caso, son necesarios más estudios en el futuro con los que validar estos primeros hallazgos e incorporar nuevas variables con el fin de conocer mejor otras posibles fuentes de varianza.

Por último, el examen de la validez mediante el análisis del FDI asociado al sexo y a las condiciones de vida reveló problemas de invarianza métrica de la escala. Al igual que otros autores (p. ej., Victor, Scambler \& Bond, 2009), el funcionamiento diferencial asociado al sexo dejó entrever distintas formas de interacción social y de experimentar la soledad: en los hombres, la soledad parece depender de la percepción de relaciones insuficientes con amigos, mientras que en las mujeres la soledad se vive como una emoción negativa resultante de la falta de personas próximas. Sin embargo, el principal problema de validez de la escala se debe al funcionamiento diferencial de los ítems debido a factores contextuales. En España, a diferencia de lo que ocurre en Holanda, el estilo de vida está más orientado a la familia (Hank, 2007; Saraceno, 2010) y el apoyo familiar que esta proporciona es distinto (Fokkema \& Liefbroer, 2008; Sánchez, De Jong Gierveld \& Buz, 2009a). Según los resultados del presente trabajo, los que viven solos se sienten más solos que los que viven acompañados y experimentan la soledad como una sensación de vacío resultante de la falta de personas queridas alrededor (normalmente cónyuge e hijos). Sin embargo, en Holanda, la experiencia de soledad es la misma para los que viven solos y para los que viven acompañados (Sánchez, De Jong Gierveld \& Buz, 2009b), quizás porque vivir solo está muy aceptado culturalmente y no tiene connotaciones negativas. Aunque según De Jong Gierveld y Van Tilburg (2010), la sensación de vacío, los sentimientos de abandono o la ausencia de personas íntimas son muy importantes en la experiencia de soledad, en España, su importancia parece depender del género y de la forma de vida. En conclusión, las amenazas a la validez, anteriormente comentadas, son evidentes y habrán de ser tenidas en cuenta en el uso de esta escala.

En el mismo sentido, la validez convergente y la discriminante presentan la dirección esperada, aunque son moderadas. De acuerdo con el modelo conceptual de la escala, la experiencia de soledad tiene un carácter no deseable en el que predominan las emociones negativas y la falta de satisfacción con la vida. Sin embargo, la moderada asociación encontrada entre estos constructos nos hace pensar que su peso es menor en personas mayores españolas que en otras culturas. De acuerdo con Padilla, Gómez, Hidalgo y Muñiz (2007), a nivel clínico y epidemiológico, las consecuencias de estas carencias detectadas en la validez deberán ser investigadas con el objeto de poder disponer de instrumentos con las mejores garantías psicométricas.

\section{Referencias}

Abad, J. F., Olea, J., Ponsoda, V. \& García, C. (2011). Medición en ciencias sociales y de la salud. Madrid: Síntesis.

Bartlett, H., Warbutton, J., Lui, C. W. \& Peach, L. (2008). Cross-government project to reduce social isolation of older people (Report on the findings from the evaluation of the demonstration projects. Project Phase 4). Brisbane, Australia: University of Queensland, Australasian Centre on Ageing.

Benjamini, Y. \& Hochberg, Y. (1995). Controlling the false discovery rate: A practical and powerful 
approach to multiple testing. Journal of the Royal Statistical Society, 57(1), 289-300.

De Jong Gierveld, J. (1987). Developing and testing a model of loneliness. Journal of Personality and Social Psychology, 53(1), 119-128.

De Jong Gierveld, J. \& Kamphuis, F. H. (1985). The development of a Rasch-type loneliness scale. Applied Psychological Measurement, 9(3), 289-299.

De Jong Gierveld, J. \& Van Tilburg, T. G. (2010). The De Jong Gierveld short scales for emotional and social loneliness: Tested on data from 7 countries in the UN generations and gender surveys. European Journal on Ageing, 7(2), 121-130.

De Jong Gierveld, J. \& Van Tilburg, T. G. (2011). Manual of the Loneliness Scale 1999 (Actualización de la versión impresa). Recuperado el 28 de septiembre de 2011, de http://home.fsw.vu.nl/TG.van. Tilburg/manual_loneliness_scale_1999.html

Diener, E., Emmons, R., Larsen, J. \& Griffin, S. (1985). The Satisfaction with Life Scale. Journal of Personality Assessment, 49(1), 71-75.

Dykstra, P., Van Tilburg, T. G. \& De Jong Gierveld, J. (2005). Changes in older adult loneliness: Results from a seven-year longitudinal study. Research on Aging, 27(6), 725-747.

Dykstra, P. A. (2009). Older adult loneliness: Myths and realities. European Journal on Ageing, 6(2), 91-100.

Embretson, S. E. \& Reise, S. P. (2000). Item response theory for psychologists. Mahwah, NJ: Erlbaum.

Ferrando, P. J. \& Anguiano-Carrasco, C. (2010). El análisis factorial como técnica de investigación en Psicología. Papeles del Psicólogo, 31(1), 18-33.

Fokkema, T. \& Liefbroer, A. C. (2008). Trends in living arrangements in Europe: Convergence or divergence? Demographic Research, 19(36), 1351-1418.

Hank, K. (2007). Proximity and contacts between older parents and their children: A European comparison. Journal of Marriage and Family, 69(1), 157-173.

Hu, L. T. \& Bentler, P. M. (1998). Fit indices in covariance structure modeling: Sensitivity to underparameterized model misspecification. Psychological Methods, 3(4), 424-453.

Instituto de Mayores y Servicios Sociales. (2008). Informe 2008. Las personas mayores en España. Datos estadísticos y por comunidades autónomas (Vol. 1). Madrid: Autor.

Jöreskog, K. G. (1977). Factor analysis by least-squares and maximum-likelihood methods. En K. Enslein, A. Ralston \& H. S. Wilf (Eds.), Statistical methods for digital computers (Vol. 3, pp 125-153). New York: Wiley.

Kunts, M., Bogaerts, S. \& Winkel, F. W. (2010). Domestic violence and mental health in a Dutch community sample: The adverse role of loneliness. Journal of Community and Applied Social Psychology, 20(5), 419-425.

Linacre, J. M. \& Wright, B. D. (2000). WINSTEPS: Multiple-choice, rating scale, and partial credit Rasch analysis [Computer program]. Chicago: MESA Press.

Lorenzo, L. \& Castejón, P. (2008). Formas de convivencia, relaciones personales y la experiencia de envejecer. En IMSERSO (2008), Informe 2008. Las personas mayores en España. Datos estadísticos y por comunidades autónomas (Vol. 1, pp. 225-271). Madrid: IMSERSO.

Lorenzo-Seva, U. \& Ferrando, P. J. (2006). FACTOR: A computer program to fit the exploratory factor analysis model. Behavioral Research Methods, Instruments and Computers, 38(1), 88-91.

Losada, A., Márquez, M., García, L., Gómez, M. A., Fernández, V. \& Rodríguez, E. (2012). Loneliness and mental health in a representative sample of community-dwelling Spanish older adults. The Journal of Psychology. Interdisciplinary and Applied, 146(3), 277-292.

Mislevy, R. J. \& Bock, R. D. (1990). BILOG 3: Item analysis and test scoring with binary logistic models. Mooresville, IN: Scientific Software.

Moorer, P. \& Suurmeijer, T. P. (1993). Unidimensionality and cumulativeness of the Loneliness Scale using Mokken Scale Analysis for polychotomous items. Psychological Reports, 73(3), 1324-1326.

Muñiz, J. (2004). La validación de los tests. Metodología de las Ciencias del Comportamiento, 5(2), 121-141.

Padilla, J. L., Gómez, J., Hidalgo, M. D. \& Muñiz, J. (2007). Esquema conceptual y procedimientos para analizar la validez de las consecuencias del uso de los test. Psicothema, 19(9), 173-178. 
Pons, D., Atienza, F. L., Balaguer, I. \& García, M. (2002). Propiedades psicométricas de la Escala de Satisfacción con la Vida en personas de tercera edad. Revista Iberoamericana de Diagnóstico y Evaluación Psicológica, 13(1), 71-82.

Prieto, G. \& Delgado, A. R. (2003). Análisis de un test mediante el modelo de Rasch. Psicothema, 15(1), 94-100.

Prieto, G. \& Delgado, A. R. (2010). Fiabilidad y validez. Papeles del Psicólogo, 31(1), 67-74.

Prieto, M. E., Forjaz, M. J., Fernández, G., Rojo, F. \& Martínez, P. (2011). Factors associated with loneliness of noninstitucionalized and institucionalized older adults. Journal of Aging and Health, 23(1), 177-194.

Rokach, A., Orzeck, T., Cripps, J., Lackovic-Grgin, K. $\&$ Penezic, Z. (2001). The effects of culture on the meaning of loneliness. Social Indicators Research, $53(1), 17-31$.

Sánchez, M. M. (2009). Determinantes sociales de la soledad en las personas mayores españolas: dar y recibir apoyo en el proceso de envejecer. Tesis doctoral no publicada, Universidad de Salamanca, Salamanca, España.

Sánchez, M. M., De Jong Gierveld, J. \& Buz, J. (2009a). Network characteristics, support exchange, and loneliness among Spanish and Dutch older adults. Proceedings of the 62nd GSA Annual Scientific Meeting. The Gerontologist, 49 (S2), 196-196.

Sánchez, M. M., De Jong Gierveld, J. \& Buz, J. (2009b). Living alone, social isolation and loneliness in Spain and The Netherlands. Proceedings of the 19th IAGG World Congress of Gerontology and Geriatrics, Paris, France, 7-9 julio 2009. Journal of Nutrition Health 8 Aging, 13(S1), 337-337.

Sánchez, M. M., De Jong Gierveld, J. \& Buz, J. (2012, 21 de septiembre). Loneliness and the exchange of social support among older adults in Spain and the Netherlands. Ageing 83 Society. doi:10.1017/ S0144686X12000839

Sandín, B., Chorot, P., Lostao, L., Joiner, T. E., Santed, M. A. \& Valiente, R. M. (1999). Escalas PANAS de afecto positivo y negativo: validación factorial y convergencia transcultural. Psicothema, 11(1), $37-51$.
Saraceno, C. (2010). Social inequalities in facing oldage dependency: A bi-generational perspective. Journal of European Social Policy, 20(1), 32-44.

Scharf, T. \& De Jong Gierveld, J. (2008). Loneliness in urban neighbourhoods: An Anglo-Dutch comparison. European Journal on Ageing, 5(2), 103-115.

Tilvis, R. S., Laitala, V., Routasalo, P. E. \& Pitkälä, K.H. (2011, 22 de febrero). Suffering from loneliness indicates significant mortality rates of older people. Journal of Aging Research, 4. doi: 10.4061/2011/534781

Van Baarsen, B., Snijders, T. A., Smit, J. H. \& Knipscheer, K. P. M. (1999). Do personal conditions and circumstances surrounding partner loss explain loneliness in newly bereaved older adults. Ageing and Society, 19(4), 441-469.

Van Baarsen, B., Snijders, T. A., Smit, J. H. \& Van Duijn, M. A. (2001). Lonely but not alone: Emotional isolation and social isolation as two distinct dimensions of loneliness in older people. Educational and Psychological Measurement, 61(1), 119-135.

Van Tilburg, T. G. (1988). Obtained and desired social support in association with loneliness. Disertación doctoral no publicada, Vrije Universiteit Amsterdam, The Netherlands.

Van Tilburg, T. G. \& De Leeuw, E. (1991). Stability of scale quality under various data collection procedures: A mode comparison on the De Jong Gierveld Loneliness Scale. International Journal of Public Opinion Research, 3(1), 69-85.

Van Tilburg, T. G., Havens, B. \& De Jong Gierveld, J. (2004). Loneliness among older adults in the Netherlands, Italy and Canada: A multifaceted comparison. Canadian Journal of Aging, 23(2), 169-180.

Victor, C., Scambler, S. \& Bond, J. (2009). The social world of older people: Understanding loneliness and social isolation in later life. Maidenhead, UK: Open University Press.

Victor, C., Scambler, S., Bond, J. \& Bowling, A. (2000). Being alone in later life: Loneliness, social isolation and living alone. Reviews in Clinical Gerontology, 10(4), 407-417.

Walker, A. (2005). A European perspective on quality of life in old age. European Journal on Ageing, 2(1), 2-12. 
Watson, D., Clark, L. \& Tellegen, A. (1988). Development and validation of brief measures of positive and negative affect: The PANAS Scales. Journal of Personality and Social Psychology, 54(6), 1063-1070.

Weiss, R. S. (1973). Loneliness: The experience of emotional and social isolation. Cambridge, MA: MIT Press.
Wright, B. D. \& Douglas, G. A. (1976). Rasch item analysis by hand (Research Memorandum No. 21). Chicago, IL: University of Chicago, Department of Education, Statistical Laboratory.

Zammuner, V. L. (2008). Italian's social and emotional loneliness: The results of five studies. International Journal of Social Sciences, 3(2), 108-120. 
